\title{
The Alignment Effect in Compact Steep Spectrum Radio Sources
}

W. H. de Vries, C. P. O’Dea, \& S. A. Baum

STScI, 3700 San Martin Drive, Baltimore, MD 21218, U.S.A.

P. D. Barthel

Kapteyn Astronomical Institute, NL-9700 AV, Groningen, The Netherlands

Abstract. Using Hubble Space Telescope WFPC2 images of 3CR compact steep spectrum (CSS) radio sources and literature radio maps makes detailed comparison between optical and radio structures possible. All CSS sources for which the optical and radio orientation can reliably be measured, display good alignment between the optical and radio emission. This alignment is observed down to the lowest redshift in the sample, at $z \sim 0.1$. Optical emission from CSS galaxies is found to be dominated by this aligned component, especially at higher redshifts. Both the alignment and the relative brightness of this component indicate that CSS sources have large amounts of matter co-spatial with the radio structure. In order to assess the nature of the aligned light, we calculated line emission contamination in the passband using ground based spectra. From this we can conclude that at least in some sources (notably 3C 213.1, 3C 346, and 3C 380) line emission is not important. The detailed opticalradio correspondence makes optical synchrotron the most likely mechanism for these three sources.

\section{Introduction}

Compact steep-spectrum radio sources (Fanti et al. 1990) may form the intermediate stage in proposed radio source evolution from the very compact $(<1 \mathrm{kpc})$ gigahertz peaked spectrum radio sources (O'Dea et al. 1991), via the CSS, to the large scale $(>20 \mathrm{kpc}$ ) FR II classical double radio sources. The alignment between optical and radio structures has been detected in the large sources (e.g., Chambers et al. 1987; McCarthy et al. 1987), so an alignment in the smaller CSS sources would be consistent with the evolutionary scenario. With the advent of the Hubble Space Telescope a comparison between optical and radio structures with similar resolution ( $\sim 45$ milliarcseconds) is possible for the first time.

Table 1. Classification of CSS alignment

\begin{tabular}{llllllll}
\hline \hline Category & Total & & Quasars & \multicolumn{3}{c}{ Galaxies } \\
\hline a) detailed corresp. & 3 & 3C 380 & & & 3C 213.1 & 3C 346 \\
b) global alignment & 13 & 3C 138 & 3C 277.1 & 3C 455 & 3C 49 & 3C 67 & 3C 216 \\
& & & & & 3C 237 & 3C 266 & 3C 268.3 \\
& & & & & 3C 299 & 3C 303.1 & 3C 305.1 \\
& & & & & & & \\
c) undetermined & 14 & 3C 43 & 3C 147 & 3C 186 & 3C 93.1 & 3C 119 & 3C 258 \\
& & 3C 190 & 3C 191 & 3C 286 & & & \\
& & 3C 287 & 3C 298 & 3C 309.1 & & & \\
\hline
\end{tabular}

Overlaying our F702W (a broad red filter) images with literature radio data (mostly VLA and MERLIN maps), we detect a strong alignment effect in CSS 
sources. It can be classified as: (1) detailed correspondence; if radio and optical structures spatially coincide (e.g., hotspots), (2) global alignment; if the angle between the axes is less than $20^{\circ},(3)$ no determination possible; if the source is heavily PSF dominated, or the radio map is of insufficient quality. The results are listed in Table 1, two example sources are shown in Figure 1.
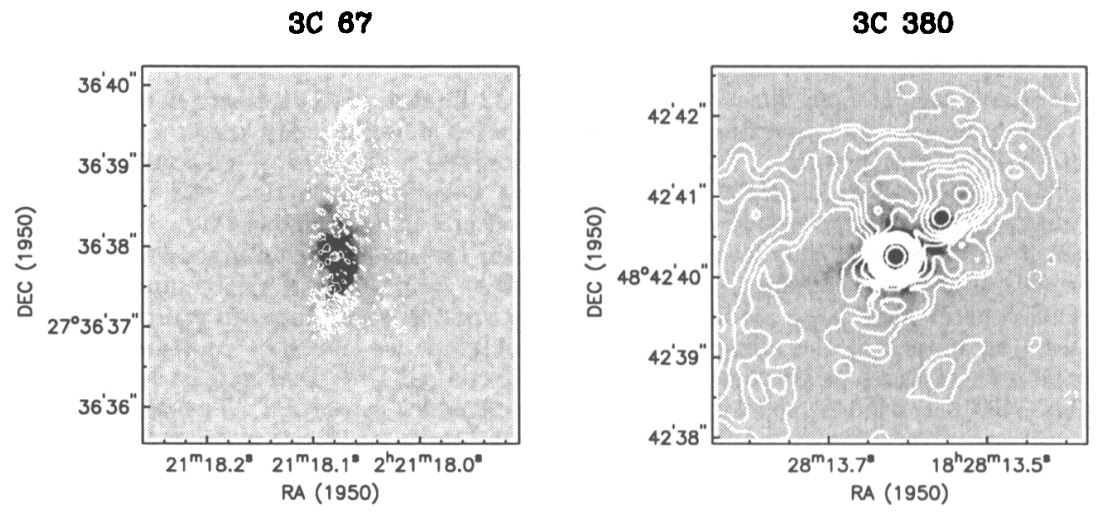

Figure 1. 3C 67 (left panel), displaying the characteristically good alignment between optical and radio structures. 3C 380 (right panel), is an example of a source with detailed correspondence.

\section{Summary}

For all CSS sources where an alignment measurement was possible, we found a good correlation between the radio and the optical emission (de Vries et al. 1997). Using ground based spectral data, we can rule out line emission as the aligned component in the sources with detailed correspondence. Those sources most likely have significant amounts of optical synchrotron emission. Follow up spectral research is needed to identify the alignment nature in the other CSS sources.

\section{References}

Chambers, K. C., Miley, G. K., \& van Breugel, W. 1987. Nature, 329, 604-606.

de Vries, W. H., et al. 1997. ApJS, 110, 191-211.

Fanti, R., et al. 1990. $A \mathscr{E} A, \mathbf{2 3 1}, 333-346$.

McCarthy, P. J., et al. 1987. $A p J$, 321, L29-33.

O'Dea, C. P., Baum, S. A., \& Stanghellini, C. 1991. ApJ, 380,66-77. 